Revista de Matemática: TeORÍA y APlicaCiones 2015 22(1) : 71-87

CIMPA - UCR ISSN: 1409-2433 (PRINT), 2215-3373 (ONLINE)

\title{
AN ADAPTIVE WAVELET-GALERKIN METHOD FOR PARABOLIC PARTIAL DIFFERENTIAL EQUATIONS
}

\section{UN MÉTODO WAVELET-GALERKIN ADAPTATIVO \\ PARA ECUACIONES DIFERENCIALES PARCIALES PARABÓLICAS}

\author{
VICTORIA VAMPA* ${ }^{*}$ MARÍA T. MARTÍN ${ }^{\dagger}$
}

Received: 25/Feb/2014; Revised: 28/Aug/2014;

Accepted: 19/Sep/2014

*Departamento de Ciencias Básicas, Facultad de Ingeniería, Universidad Nacional de La Plata, Argentina. E-Mail: victoriavampa@gmail.com

${ }^{\dagger}$ Facultad de Ciencias Exactas, Universidad Nacional de La Plata, Argentina. IFLP-CCTCONICET, C. C. 727, 1900 La Plata, Argentina. E-Mail: teremartin.map@gmail.com 


\begin{abstract}
In this paper an Adaptive Wavelet-Galerkin method for the solution of parabolic partial differential equations modeling physical problems with different spatial and temporal scales is developed. A semi-implicit time difference scheme is applied and $B$-spline multiresolution structure on the interval is used. As in many cases these solutions are known to present localized sharp gradients, local error estimators are designed and an efficient adaptive strategy to choose the appropriate scale for each time is developed. Finally, experiments were performed to illustrate the applicability and efficiency of the proposed method.
\end{abstract}

Keywords: $B$-spline; multiresolution analysis; wavelet-Galerkin.

\title{
Resumen
}

En este trabajo se desarrolla un método Wavelet-Galerkin Adaptativo para la resolución de ecuaciones diferenciales parabólicas que modelan problemas físicos, con diferentes escalas en el espacio y en el tiempo. Se utiliza un esquema semi-implícito en diferencias temporales y la estructura multirresolución de las B-splines sobre intervalo.Como es sabido que en muchos casos las soluciones presentan gradientes localmente altos, se han diseñado estimadores locales de error y una estrategia adaptativa eficiente para elegir la escala apropiada en cada tiempo. Finalmente, se realizaron experimentos que ilustran la aplicabilidad y la eficiencia del método propuesto.

Palabras clave: $B$-spline, análisis multirresolución; wavelet-Galerkin; ondeletas Galerkin.

Mathematics Subject Classification: 65M99.

\section{Introduction}

Analytical solutions for nonlinear partial differential equations which describe physical phenomena, such as the equations of fluid mechanics, are usually difficult to be obtained. In the development of numerical schemes, the use of multiresolution techniques and wavelets has become increasingly popular and wavelet-Galerkin approximations have been applied as an alternative to conventional finite element methods.

When the solution exhibits multiscale features like coarse solution in the whole domain and details near singularities, recalculation of the solution in finer meshes is needed to get the desired convergence. Adaptive refinement eliminates the need to remesh the whole domain and there are a number of papers in this 
direction where different adaptive strategies are designed and applied in solving both, ordinary and partial differential equations.

Quraishi et al. [11] applied second generation wavelets as basis in the finite element method for elastostatics problems. They developed a wavelet-Galerkin method with an adaptive scheme choosing higher density of nodes in regions where sharp change or gradient is presented. In Kumar et al. [8] a collocation method to solve singularly perturbed reaction diffusion equation of elliptic and parabolic types using cubic splines is presented and an efficient adaptive feature is performed automatically by thresholding the wavelet coefficients.

For the solution of parabolic equations in cases there exists different spatial and temporal scales as in equations modeling the formation of shock waves in compressible gas flow several numerical methods have been proposed. Vasilyev et al. [17] developed a dynamically adaptive multilevel wavelet collocation method for the solution of partial differential equations using Daubechies scaling functions. They applied the method to the solution of the Burgers equation with small viscosity and to the solution of a moving shock problem.

In other proposals, Galerkin methods are used and in deriving the computational schemes, time discretization is performed prior to wavelet-based Galerkin spatial approxi-mation. Schult and Wyld [13] employed a set of suitably selected coarse-scale scaling functions and fine scale wavelets centered at the discontinuity. Using Daubechies scaling functions, a second order time stepping scheme was applied to advance in time. Lin and Zhou [9] used a semi-implicit time difference scheme combined with wavelet interpolation based approximations. Only scaling functions were used, and coiflets were chosen. On the other hand, Kumar and Mehra [7], in 2005, developed a Taylor-generalized Euler time discretization method. Bindal et. al [2] presented a dynamically adaptive algorithm for solving PDEs where Galerkin is used to discretize spatial variables and then, a system of ordinary differential equations has to be solved. In the proposed algorithm, successive iterations result in smaller wavelet coefficients and approximations with the desired accuracy are obtained. Daubechies scaling and wavelet functions are used.

The aim of this paper is to formulate an efficient method to solve parabolic equations with scaling functions and wavelets, extending the refinement process developed in [16] for second order boundary value problems. An adaptive algorithm based on the analysis of wavelets coefficients is incorporated and allow to follow the local structures of the solution.

The novelty of our proposal is that an adaptive algorithm in each time step is implemented: once an approximation in terms of scaling functions at an initial coarse-scale is obtained, an error estimation allows to determine the 
scale $j$ necessary to achieve the required precision. And this is done at each time step. We will call this method Adaptive Wavelet-Galerkin (AWG). It provides a simple way to adapt computational refinements to local demands of the solution. High scales are used only in regions where sharp transition occurs. The method is applied to the solution of one dimensional Burgers equation with small viscosity.

The organization of this paper is as follows: In Section 2, a semi-implicit scheme to advance in time is presented to solve parabolic equations and we described the AWG method to solve boundary value problems: how to define an MRA on the interval [4], the Modified Galerkin method [14] to obtain the approximation at an initial scale in terms of scaling functions and how wavelets are used to increase the scale efficiently [16]. Finally, an error estimation is given. The algorithm to solve parabolic equations after integration in time is performed, applying the AWG method is described in Section 3. The method is tested on the one-dimensional Burgers equation with two different types of boundary conditions. Numerical results are shown in Section 4. Finally, conclusions are presented in Section 5.

\section{Adaptive Wavelet-Galerkin method}

Different physical situations are modeled with parabolic differential equations of the type

$$
u_{t}(x, t)+F(u)(x, t) u_{x}(x, t)=\epsilon u_{x x}(x, t),
$$

They are often referred to as being initial value problems in the sense we are given the state of a system at sometime $t=0$ and we require the state of the system at subsequent times. We will be concerned with numerical methods for initial value problems and the solution will be sought in the region $a \leq x \leq b$ for $t \geq 0$. Several schemes can be applied to advance in time.

We consider the following semi-implicit time stepping scheme [9]:

$$
\frac{u^{k+1}-u^{k}}{\Delta t}+F\left(u^{k}\right) \frac{\partial u^{k+1}}{\partial x}=\epsilon \frac{\partial^{2} u^{k+1}}{\partial x^{2}}
$$

where $u^{k}(x)=u(x, k \Delta t)$ and $\Delta t$ is the time step. If we denote $w(x)=$ $u^{k+1}(x)$, a second order boundary value problem (BVP) for $w$ on $[a, b]$ is obtained at each time step

$$
-w^{\prime \prime}+\frac{1}{\epsilon} F\left(u^{k}\right) w^{\prime}+\frac{1}{\epsilon \Delta t} w=\frac{1}{\epsilon \Delta t} u^{k}
$$

with the corresponding boundary conditions. 
It is known that when solving these kind of equations irregular features, singularities and steep changes arise. Consequently, procedures that can resolve varying scales in an efficient manner are required. Wavelets, with their multiresolution analysis properties [10], can be used advantageously in these types of problems.

In order to solve boundary value problems on $I=[a, b]$, multiresolution structures in $L^{2}(\mathbb{R})$ have to be restricted to $L^{2}(I)$. As is described by Chui [5], an MRA on $\mathrm{L}^{2}(\mathbb{R})$ is constructed by first identifying the subspace $V_{0}$ and the scaling function $\phi$. Then, for each $j \in \mathbb{Z}$, the family $\left\{\phi_{j, k}: k \in \mathbb{Z}\right\}$, where $\phi_{j, k}(x):=2^{j / 2} \phi\left(2^{j} x-k\right)$, is a basis of $V_{j}$. Associated with the scaling function $\phi$ there exists a function $\psi$ called the mother wavelet such that the collection $\{\psi(x-k), k \in \mathbb{Z}\}$ is a Riesz basis [5] of $W_{0}$, the orthogonal complement of $V_{0}$ in $V_{1}$. If we consider, $\psi_{j, k}(x):=2^{j / 2} \psi\left(2^{j} x-k\right)$ for each $j \in \mathbb{Z}$, the family $\left\{\psi_{j, k}: k \in \mathbb{Z}\right\}$ is a basis of $W_{j}$, the orthogonal complement of $V_{j}$ in $V_{j+1}$. It is noteworthy that wavelets would allow the refinement of the representation space taking into account that

$$
V_{j+1}=V_{j} \oplus W_{j}
$$

\subsection{An MRA on the interval}

Let us assume that the support of the scaling function $\phi(x) \in V_{0}$ is $[0, S], S \in$ $N$, and the support of the wavelet $\psi \in W_{0}$ is $[-S+1, S]$. To fix notations, let us assume that the interval is $[0,1]$. Considering $j_{0}$ such that $2^{j_{0}} \geq S$, we define for $j \geq j_{0}, \phi_{j, k}^{I}(x)=\phi_{j, k}(x) \chi_{[0,1]}(x)$ and $\widehat{V}_{j}^{I}=\operatorname{gen}\left\{\phi_{j, k}^{I}(x), 1-S \leq k \leq 2^{j}-1\right\}$ as the space of basis functions that intersect the interval $[0,1]$.

In what follows, an MRA with $B$-splines as scaling functions is considered. In its construction, orthogonality conditions are used in a way similar to when an MRA is designed in $\mathrm{L}^{2}(\mathbb{R})[10,18,16] . V_{0}$ is the subspace generated by translations of the scaling function $\varphi_{m+1}$, the $B$-spline function of order $m$, and for each $j \in \mathbb{Z}$, the family $\left\{\varphi_{m+1, j, k}=2^{j / 2} \varphi_{m+1}\left(2^{j} x-k\right): k \in \mathbb{Z}\right\}$ is a basis of $V_{j}$. These subspaces $V_{j}, j \in \mathbb{Z}$, constitute an MRA in $\mathrm{L}^{2}(\mathbb{R})([10,18])$.

In the cubic $B$-spline MRA framework $[12,15]$, let us denote scaling functions by $\varphi_{j, k}^{I}(x)=\varphi_{j, k} \chi_{[0,1]}(x)$ and $j \geq 2$ (for simplicity, the first subscript is omitted, assuming $m=3$ ). They are supported on $\left[2^{-j} k, 2^{-j}(k+4)\right]$ and are splines in $\mathbb{Z} / 2^{j}$. They are interior splines if $0 \leq k \leq 2^{j}-4$ and boundary splines if $-3 \leq k \leq-1$ and $2^{j}-3 \leq k \leq 2^{j}-1$. Denoting by $V_{j}^{I}$ the space of interior scaling functions $V_{j}^{I}=\operatorname{gen}\left\{\varphi_{j, k}^{I}, 0 \leq k \leq 2^{j}-4\right\}$, of size $2^{j}-3$ and by $\widehat{V}_{j}^{I}$ the space of scaling functions $\widehat{V}_{j}^{I}=\operatorname{gen}\left\{\varphi_{j, k}^{I},-3 \leq k \leq 2^{j}-1\right\}$, of size $2^{j}-3$ and the inclusions $V_{j}^{I} \subset \widehat{V}_{j}^{I}$ and $V_{j}^{I} \subset H_{0}^{1}$ are verified. Subspaces $\widehat{V}_{j}^{I}$ 
have dimension $2^{j}+3$ and constitute an MRA in $\mathrm{L}^{2}([0,1])$. Each subspace $\widehat{V}_{j}^{I}$ consists of piecewise polynomials of degree $m=3$ with knots in $0 \leq k / 2^{j} \leq 1$.

Motivated by the construction for the whole line, a suitable basis for the wavelet space $\widehat{W}_{j}^{I}$, the orthogonal complement of $\widehat{V}_{j}^{I}$ in $\widehat{V}_{j+1}^{I}$, has the form $\left[\widehat{\psi}_{j}^{I}\right]=\left[\widehat{\varphi}_{j+1}^{I}\right] . \widehat{G}_{j}$, where $\widehat{G}_{j}$, of size $\left(2^{j+1}+3\right) \times 2^{j}$, is a matrix such that its columns are in the null space of $\widehat{H}_{j}^{t} \cdot \widehat{P}_{j+1}^{I}$ of size $2^{j}$. As described in detail in [15, 16], $\widehat{H}_{j}$ is the two scale matrix of size $\left(2^{j+1}+3\right) \times\left(2^{j}+3\right)$, such that the relation $\left[\widehat{\varphi}_{j}^{I}\right]=\left[\widehat{\varphi}_{j+1}^{I}\right] . \widehat{H}_{j}$ is satisfied, while $\widehat{P}_{j}^{I}=\left[\widehat{\varphi}_{j}^{I}\right]^{t} \cdot\left[\widehat{\varphi}_{j}^{I}\right], \widehat{P}_{j}^{I} \in \mathbb{R}^{\left(2^{j}+3\right) \times\left(2^{j}+3\right)}$ is the Grammian matrix, associated to the bases $\left[\widehat{\varphi}_{j}^{I}\right]$.

Interior wavelets are not modified, the same as scaling functions: only bases corresponding to the edges are different and are adequately designed (see [4]). Denoting by $W_{j}^{I}$ the orthogonal complement of $V_{j}^{I}$ in $V_{j+1}^{I}$ of size $2^{j}$, the construction of a basis for $W_{j}^{I}$ is analogous to the method described above for $\widehat{W}_{j}^{I}$, taking into account that $\operatorname{dim}\left(W_{j}^{I}\right)=\operatorname{dim}\left(\widehat{W}_{j}^{I}\right)=2^{j}$.

\subsection{Modified Galerkin method for a boundary value. Problem}

For the linear BVP on an interval, $L u=-u^{\prime \prime}(x)+p(x) u^{\prime}(x)+q(x) u(x)=$ $f(x)$, where $p(x), q(x)$ and $f(x)$ are continuous functions on $I$ and $u$ is a function in certain space $V$, it is known that the corresponding variational formulation is to seek $u \in V$, such that $a(u, v)=\langle f, v\rangle, \quad \forall v \in V$, where:

$$
a(u, v)=\int_{0}^{1}\left(u^{\prime}(x) v^{\prime}(x)+p(x) u^{\prime}(x) v(x)+q(x) u(x) v(x)\right) d x,
$$

for $u$ and $v \in V^{0} \subset L^{2}(I)$, the subspace of functions with homogeneous boundary conditions, in case Dirichlet boundary conditions are considered.

Let us assume that we choose an approximate solution $\tilde{u}$ of the form $\tilde{u}=$ $\sum_{k=1}^{N} \alpha_{k} \Phi_{k}^{I}$. The substitution of this approximation in the weak formulation produces the following linear system:

$$
\sum_{k=1}^{N} \alpha_{k} a\left(\Phi_{k}, \Phi_{n}\right)=\left\langle f, \Phi_{n}\right\rangle \quad n=1,2, \ldots, N,
$$

and we arrive at the problem of solving a matrix equation, $A \alpha=b$, where $A(n, k)=a\left(\Phi_{k}, \Phi_{n}\right)$ and $b(n)=\left\langle f, \Phi_{n}\right\rangle$.

If the spaces of scaling functions, $V_{j}^{I}$ or $\widehat{V}_{j}^{I}$, described in the previous section are used for the approximation solution $\widehat{u}_{j}$, the rate of convergence is not good. In the first case the reason is that in the subspace $V_{j}^{I}$, all functions and their derivatives vanish at both ends and then, a poor approximation is provided. 
On the other hand, considering scaling functions in $\widehat{V}_{j}^{I}$ the matrix tends to be very ill conditioned when the scale $j$ is large: as we have to restrict to the interval $I$, the support of the intersection of boundary splines with the rest of scaling functions becomes smaller and the rows corresponding to equations that involve the edges are almost nulls.

These drawbacks were analyzed in recent articles $[14,15,16]$ and motivated the development of the Modified Galerkin (MG) method, which combines variational equations with a collocation scheme using both spaces $V_{j}^{I}$ and $\widehat{V}_{j}^{I}$ to construct an algebraic system to obtain $\widehat{u}_{j}$ as follows:

1. (a) Variational equations: they are are obtained from the weak formulation, considering that the approximation of the unknown function $u$ is in $\widehat{V}_{j}^{I}$ and has the form $\widehat{u}_{j}=\sum_{k=-3}^{2^{j}-1} \widehat{\alpha}_{j, k} \varphi_{j, k}^{I}$, while the test function $v$ is in $V_{j}^{I}$. This leads to a rectangular system of size $\left(2^{j}-\right.$ $3) \times\left(2^{j}+3\right)$ :

$$
\sum_{k=-3}^{2^{j}-1} \widehat{\alpha}_{k} a\left(\widehat{\varphi}_{j, k}^{I}, \varphi_{j, n}^{I}\right)=\left\langle f, \varphi_{j, n}^{I}\right\rangle \quad n=0,1, \ldots,\left(2^{j}-4\right),
$$

or in matrix form,

$$
\widehat{A}_{4, j} \widehat{\alpha}_{j}=\widehat{b}_{4, j} .
$$

(b) Collocation equations: they are obtained from the requirement that the residual should be zero at the ends of the interval and in collocations points, $2^{-j}$ and $1-2^{-j}$,

$$
\begin{aligned}
u^{\prime \prime}(0)+p(0) u^{\prime}(0)+q(0) u(0) & =f(0) \\
u^{\prime \prime}\left(2^{-j}\right)+p\left(2^{-j}\right) u^{\prime}\left(2^{-j}\right)+q\left(2^{-j}\right) u\left(2^{-j}\right) & =f\left(2^{-j}\right) \\
u^{\prime \prime}\left(1-2^{-j}\right)+p\left(1-2^{-j}\right) u^{\prime}\left(1-2^{-j}\right) & \\
+q\left(1-2^{-j}\right) u\left(1-2^{-j}\right) & =f\left(1-2^{-j}\right) \\
u^{\prime \prime}(1)+p(1) u^{\prime}(1)+q(1) u(1) & =f(1) .
\end{aligned}
$$

(c) Boundary conditions: are obtained from the requirement that the solution satisfies the boundary conditions,

$$
\begin{array}{rcc}
\widehat{\alpha}_{-3} \varphi_{4, j,-3}^{I}(0)+\widehat{\alpha}_{-2} \varphi_{4, j,-2}^{I}(0)+\widehat{\alpha}_{-1} \varphi_{4, j,-1}^{I}(0) & =0 \\
\widehat{\alpha}_{2^{j}-2} \varphi_{4, j, 2^{j}-2}^{I}(1)+\widehat{\alpha}_{2^{j}-1} \varphi_{4, j, 2^{j}-1}^{I}(1)+\widehat{\alpha}_{2^{j}} \varphi_{4, j, 2^{j}}^{I}(1) & =0 .
\end{array}
$$


2. Approximate solution in $V_{j}^{I}$ : Solving the square algebraic system $2^{j}+3$ coefficients $\tilde{\alpha}_{j k}$ are obtained. Then we have to project $\widehat{u}_{j}$ in order to get the approximate solution $\tilde{u}_{j}$,

$$
\tilde{u}_{j}=\mathrm{P}_{\mathrm{V}_{\mathrm{j}}^{\mathrm{I}}}\left(\widehat{\mathrm{u}}_{\mathrm{j}}\right)=\sum_{\mathrm{k}=0}^{2^{\mathrm{j}}-4} \widehat{\alpha}_{\mathrm{j}, \mathrm{k}} \varphi_{4, \mathrm{j}, \mathrm{k}} .
$$

It should be noted that the matrix of equation (8) has a Toeplitz structure and that the final algebraic system that corresponds to the MG method has a band matrix, as sparse as in the case of considering interior basis functions only, but different at the top and at the bottom. Consequently, numerical solutions can be computed efficiently $[14,15]$. To calculate the matrix elements in equation (8) the following convolution properties of $B$-spline scaling functions were used [14]:

$$
\begin{array}{rlc}
\left\langle\varphi_{m+1, j, l}, \varphi_{m+1, j, k}\right\rangle & = & \varphi_{2(m+1)}(m+1+l-k) \\
\left\langle\varphi_{m+1, j, l}, \varphi_{m+1, j, k}^{\prime}\right\rangle & = & 2^{j} \varphi_{2(m+1)}^{\prime}(m+1+l-k) \\
\left\langle\varphi_{m+1, j, l}, \varphi_{m+1, j, k}^{\prime \prime}\right\rangle & = & -2^{2 j} \varphi_{2(m+1)}^{\prime \prime}(m+1+l-k),
\end{array}
$$

and then, taking into account equation (7), they have the following form:

$$
\begin{aligned}
\widehat{A}_{m+1, j}(n, k)=- & 2^{2 j} \varphi_{2(m+1)}^{\prime \prime}(m+1+n-k)+ \\
& 2^{j} p_{j}(n, k) \varphi_{2(m+1)}^{\prime}(m+1+n-k)+ \\
& q_{j}(n, k) \varphi_{2(m+1)}(m+1+n-k),
\end{aligned}
$$

where $\varphi_{2(m+1)}$ is the $B$-spline of order $2 m+1$ and $\widehat{b}_{m+1, j}(n)=\left\langle f, \varphi_{j, n}^{I}\right\rangle$, for $0 \leq n, k \leq 2^{j}-4$.

\subsubsection{Approximation error analysis}

To analyze the approximation error in scale $j$ using the Modified Wavelet-Galerkin Method, it is important to take into account that $\widehat{u}_{j}$ is not the solution of a pure variational problem in $\widehat{V}_{j}^{I}$, so then Céa's lemma [6] cannot be applied. However, as was demonstrated by the authors (see $[15,16]$ for details), it is possible to design a subspace $\widehat{U}_{j}^{I}$ of $\widehat{V}_{j}^{I}$ in such a way that the approximation $\widehat{u}_{j}$ is the solution of a classic variational problem in $\widehat{U}_{j}^{I}$. Then, the following error estimation is obtained:

$$
\left\|u-\widehat{u}_{j}\right\|_{\widehat{V}_{j}^{I}}^{2} \leq \frac{C}{\gamma} i n f_{v \in \widehat{U}_{j}^{I}}\|u-v\|_{\widehat{V}_{j}^{I}}^{2}
$$


where $C$ and $\gamma$ are constants corresponding to continuity and coercivity of the bilinear form $a$, respectively.

From (16) it is derived that the solution obtained with the Modified Galerkin Method minimizes norm error (with a constant factor) and converges to the exact solution as the scale $j$ increases.

It is demonstrated in [12] that the interpolatory cubic spline function $S_{h}$, which coincides with a smooth function $u \in C^{4}$ with uniform spacing $h$, satisfies:

$$
\left\|u-S_{h}\right\|_{H^{1}}^{2} \leq \frac{35}{24} h^{4}\|u\|_{\infty} .
$$

Finally, and as a consequence of the above results, the following bound is valid for the approximation error:

$$
\left\|u-\widehat{u}_{j}\right\|_{L^{2}}^{2} \leq \bar{C}\left(\frac{1}{2^{j}}\right)^{4}
$$

\subsection{Using wavelets to increase the scale}

Once $\widehat{u}_{j}$ at scale $j$ is obtained, an error estimate of the approximation in $\widehat{V}_{j}^{I}$ may indicate the convenience of increasing the scale. Instead of repeating the process described previously, an attractive strategy consists of improving the approximation recursively using wavelets. In this way, the MRA structure is exploited and large computational savings could be achieved.

Let us consider the following expansion for $\widehat{u}_{j+1} \in \widehat{V}_{j+1}^{I}$ :

$$
\widehat{u}_{j+1}=\sum_{k=-3}^{-1} \widehat{\alpha}_{j+1, k} \varphi_{j+1, k}^{I}+\sum_{k=0}^{2^{j+1}-4} \widehat{\alpha}_{j+1, k} \varphi_{j+1, k}^{I}+\sum_{k=2^{j+1}-3}^{2^{j+1}-1} \widehat{\alpha}_{j+1, k} \varphi_{j+1, k}^{I} .
$$

Taking into account that $\sum_{k=0}^{2^{j+1}-4} \widehat{\alpha}_{j+1, k} \varphi_{j+1, k}^{I} \in V_{j+1}^{I}$ and the space orthogonality relation, (equation (4)), equation (19) can be rewritten using another basis of $\widehat{V}_{j+1}^{I}$ :

$$
\widehat{u}_{j+1}=\sum_{k=-3}^{-1} \widehat{\alpha}_{j+1, k} \varphi_{j+1, k}^{I}+\sum_{k=0}^{2^{j}-4} \beta_{j, k} \varphi_{j, k}^{I}+\sum_{k=1}^{2^{j}} \xi_{j, k} \psi_{j, k}^{I}+\sum_{k=2^{j+1}-3}^{2^{j+1}-1} \widehat{\alpha}_{j+1, k} \varphi_{j+1, k}^{I} .
$$

Then, solving the following variational equations:

$$
\begin{aligned}
\left\langle L \widehat{u}_{j+1}, \varphi_{j, n}^{I}\right\rangle & =\left\langle f, \varphi_{j, n}^{I}\right\rangle & & 0 \leq n \leq 2^{j}-4 \\
\left\langle L \widehat{u}_{j+1}, \psi_{j, n}^{I}\right\rangle & =\left\langle f, \psi_{j, n}^{I}\right\rangle & & 1 \leq n \leq 2^{j}
\end{aligned}
$$


with the six additional equations corresponding to both edges, the coefficients of $\widehat{u}_{j+1}$ can be obtained. Considering that $\widehat{u}_{j+1}=\widehat{u}_{j}+\left[\widehat{u}_{j+1}-\widehat{u}_{j}\right]=\widehat{u}_{j}+\widehat{v}_{j}$, the increment $\widehat{v}_{j} \in \widehat{V}_{j+1}$ can be expressed as:

$$
\widehat{v}_{j}=\sum_{k=-3}^{2^{j+1}-1} \gamma_{j+1, k} \widehat{\varphi}_{j+1, k}^{I}=\left[\widehat{\varphi}_{j+1}^{I}\right] \cdot\left[\gamma_{j+1}\right],
$$

and replacing $\widehat{u}_{j+1}$ into equation (21), yields the following important result.

Lemma 1 The increment $\widehat{v}_{j}$ satisfies the orthogonality condition, $\left\langle L \widehat{v}_{j}, \varphi_{j, n}^{I}\right\rangle=$ $0,0 \leq n \leq 2^{j}-4$.

Due to this lemma the total number of unknowns is significantly decreased from $\left(2^{j+1}+3\right)$ to $\left(2^{j}+6\right)$ as a consequence of the following theorem.

Theorem 1 There exists a matrix $N_{j}$ of size $\left(2^{j+1}+3\right) \times\left(2^{j}+6\right)$, recursive and of simple structure and a vector $\widehat{\alpha}_{j+1}$ of length $\left(2^{j}+6\right)$ such that the increment coefficients in the basis of scaling functions of $\widehat{V}_{j+1}$ are $\left[\gamma_{j+1}\right]=N_{j}\left[\widehat{\alpha}_{j+1}\right]$.

In conclusion, once the approximation $\widehat{u}_{j}$ is obtained, the scale can be increased efficiently using the AWG method (for details see [16]), which consists of solving for the increment $\widehat{v}_{j}, 2^{j}$ variational equations,

$$
\left\langle L \widehat{v}_{j}, \psi_{j, n}^{I}\right\rangle=\left\langle f-L \widehat{u}_{j}, \psi_{j, n}^{I}\right\rangle,
$$

and imposing six equations corresponding to both edges.

Using the property described in the theorem above, large computations savings can be achieved since the effort required to advance form scale $j$ to $j+1$ using scaling functions is twice the effort using wavelets and solving equation (24).

\subsubsection{Adaptivity: error estimate and refinement criteria}

In this section, an error estimate for the approximate solution is obtained. Taking the norm of the increment in equation (23), the following expression is obtained for each scale:

$$
\left\|\widehat{v}_{j}\right\|_{2}^{2} \leq C_{j} \sum_{k=-3}^{2^{j+1}-1}\left|\gamma_{j+1, k}\right|^{2} .
$$

As the functions $\varphi_{j, k}^{I}$ constitute a Riesz basis of $\widehat{V}_{j+1}^{I}$, equation (25) is verified for certain constants $C_{j}$, with $C_{j} \leq C$, for all $j$, see [5]. Then, the increment coefficients constitute a natural expression for the error in the $L^{2}$ norm and $\sum_{k=-3}^{2^{j+1}-1}\left|\gamma_{j+1, k}\right|^{2}$ can be used as an error estimate. 
Once such an estimate has been made, one can decide whether the approximation is satisfactory or whether further refinement of the solution is necessary. In the following section, the adaptive scheme for parabolic equations using this refinement criteria is described.

\section{AWG algorithm}

Let us summarize the iterative refinement algorithm proposed to solve equation (3):

\section{- Step 1}

Choose $\Delta t$ and set an initial coarse scale $j . \quad u^{0}(x)=u(x, 0)$ (initial condition, $t=k \Delta t, k=0$ ).

\section{- Step 2}

Solve the linear system using the Modified Wavelet-Galerkin Method (Sec-

tion 2.2) considering $p(x)=\frac{1}{\epsilon} F\left(u^{k}(x)\right), q(x)=\frac{1}{\epsilon \Delta t}$ and $f(x)=\frac{1}{\epsilon \Delta t} u^{k}(x)$. $\widehat{u}_{j}^{k+1}=\sum_{k=-3}^{2^{j}-1} \widehat{\alpha}_{j, k} \varphi_{j, k}^{I}$ is obtained.

- Step 3

Refine and advance in $j$ with wavelets: Find $\left[\widehat{\alpha}_{j+1}\right]$ and $\widehat{v}_{j}$ solving the system equation (24).

- Step 4

Given an adequate threshold $\varepsilon$, IF $\left\|\widehat{v}_{j}\right\|_{2}^{2}<\varepsilon$, a good approximation $\widehat{u}_{j_{w}}^{k+1}$ is obtained ( $j_{w}$ is the refined scale) Go TO Step 6,

IF NOT, GO TO Step 5.

- Step 5 $\widehat{u}_{j+1}=\widehat{u}_{j}+\widehat{v}_{j}, j=j+1$ go back to Step 3 .

- Step 6

$t=t+\Delta t(k=k+1)$. If $t=t_{\text {final }}$, STOP. Otherwise, find $u_{j}^{k}=$ $\operatorname{Proj}\left(\widehat{u}_{j_{w}}^{k}\right)$ on to $V_{j}$ and REPEAT steps $2-5$.

\section{Remark}

In Step 6, $\widehat{u}_{j_{w}}^{k}$ for $t$ is obtained at scale $j_{w}>j$. As in Step 2, $u_{j}^{k}$ is needed to solve the MG method but in scale $j$, the projection $\operatorname{Proj}\left(\widehat{u}_{j_{w}}^{k}\right)$ on to $V_{j}$ is used leading to big savings in computer time. This projection is calculated by multiplying by two scale matrices $\widehat{H}_{j}$ from $j$ to $j_{w}$. 


\section{Numerical results}

In this section experiments are presented to demonstrate the capability of the proposed method: the Burgers equation is solved with two different types of boundary and initial conditions.

It is important to point out the advantages of the proposed method in choosing automatically the scale needed to get the required accuracy for each time step: the algorithm performs spacial adaptivity letting the time step fixed. In addition, as it was mentioned before, algebraic systems are efficiently solved because matrices are sparse, well conditioned and with Toeplitz structure.

\subsection{Burgers equation}

In 1939, J. M. Burgers [3] simplified the Navier-Stokes equation and obtained the following nonlinear equation:

$$
u_{t}(x, t)+u(x, t) u_{x}(x, t)=\epsilon u_{x x}(x, t)+F(x, t),
$$

known as the Burgers equation, where $\epsilon$ is the viscosity, frequently considered without external force $F(x, t)$.

In general, nonlinear equations cannot be solved analytically. But in this case, the Cole-Hopf transformation [19] turns the nonlinear Burgers equation into the linear heat conduction equation. Since the heat equation is explicitly solvable in terms of the so-called heat kernel, a general solution of the Burgers equation can be obtained. This is of importance because it allows one to compare numerically obtained solutions of the nonlinear equation with the exact ones, which is very useful to investigate the quality of the applied numerical schemes. Furthermore, the Burgers equation still has interesting applications in physics and astrophysics.

\section{- Problem 1:}

For the first test problem we consider equation (26) for $0 \leq x \leq 1$.

$$
u_{t}(x, t)+u(x, t) u_{x}(x, t)=\frac{10^{-2}}{\pi} u_{x x}(x, t) .
$$

The following Dirichlet boundary conditions $u(0, t)=u(1, t)=0$ and initial condition, $u^{0}(x)=u(x, 0)=-\sin (\pi 2 x-1)$ are used. We have solved this problem with a fixed time integration step, $\Delta t=0.0025$, an initial scale $j=j_{0}=6$ and $t_{\text {final }}=0.1875$. 
For the initial condition considered, the sine curve steepens as time advances, leading to a stationary discontinuity at $x=0.5$.

First, in Figure 1 we present numerical results obtained with the proposed algorithm but using a fixed scale $j$ for all time steps, without adaptivity. The oscillations are due to the fact that $j$ is small and is not sufficient to resolve the large gradient that occurs at $x=0.5$. Secondly, in Figure 2 numerical solutions are shown applying the adaptivity strategy proposed. $\varepsilon=0.510^{-2}$ is the threshold for the coefficients of the increment $\widehat{v}_{j}$ $\left(\widehat{u}_{j+1}=\widehat{u}_{j}+\widehat{v}_{j}\right)$ that was used as termination criterion. The scale take increasing values $6 \leq j_{w} \leq 10$ as time advance, and is maximun for the time the solution present a sharp gradient.

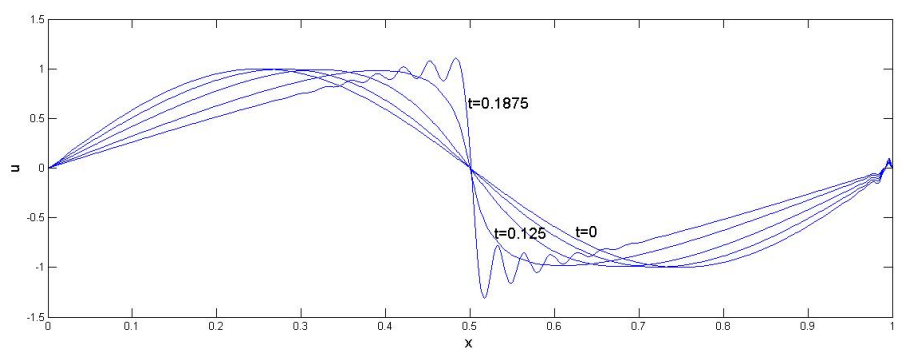

Figure 1: Evolution of Burgers equation solution $(0 \leq t \leq 0.25)$.

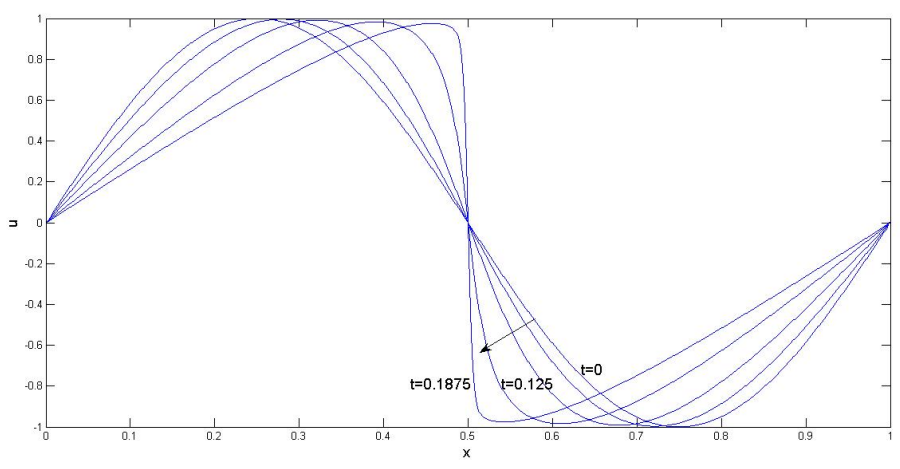

Figure 2: Evolution of Burgers equation solution $(0 \leq t \leq 0.25)$.

It should be noted that the value of the threshold must be chosen appropriately, in order computational savings could be achieved. For a smaller 
threshold $\varepsilon=0.510^{-4}$ the scale $j=10$ is needed and no adaptivity is performed.

In Figure 3 we present the approximation error versus $x$, for $t=4 \Delta t$ and $t=15 \Delta t$, in both cases taking $j=10$. Adequate scale factors were used to make possible the comparison. For the second time value increment, coefficients are concentrated around the singularity located at $x=0.5$ (See Figures 1 and 2).
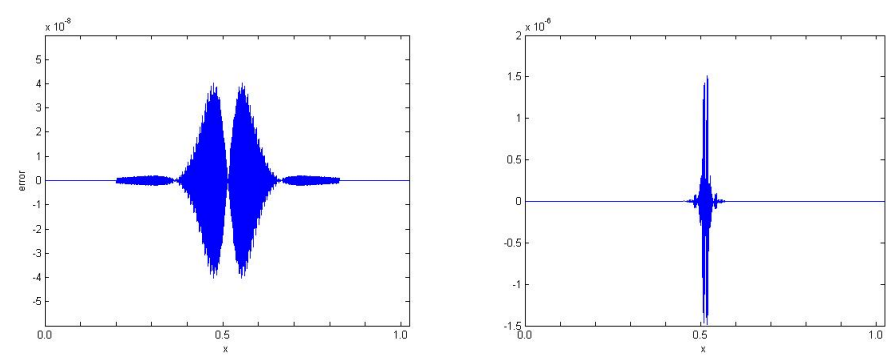

Figure 3: The approximation error versus $x$ (with knots in $k / 2^{10}, k=0, \ldots, 2^{10}$ ). Left for $t=0.01$ and right for $t=0.0375$.

- Problem 2:

As a second test problem, we consider equation (26) for $0 \leq x \leq 1$

$$
u_{t}(x, t)+u(x, t) u_{x}(x, t)=(0.002) u_{x x}(x, t)
$$

with the following mixed boundary conditions $u_{x}(0, t)=0$ and $u(1, t)=$ 1 and $u^{0}(x)=u(x, 0)=e^{-8(1-x)}$ as the initial condition.

In Figure 4 we present numerical results obtained with the proposed algorithm. In this case, the fix integration time step is $\Delta t=0.015$, an initial scale $j=j_{0}=6, t_{\text {final }}=1.515$. A quasi shock starting at $t=0.03$ and fully developed at $t=0.2$ is shown. $\varepsilon=0.510^{-3}$ is the threshold for the coefficients of the increment $\widehat{v}_{j}$.

In Figure 5 we present the approximation error versus $x$ for $t=10 \Delta t$ and $t=25 \Delta t$ (for $j=7$ left and $j=8$ right).

It is evident from the plots that in both problems, the adaptive algorithm is able to track the sharp changes of the solutions and that high-order accuracy can be achieved by the adaptive Wavelet-Galerkin scheme proposed. 


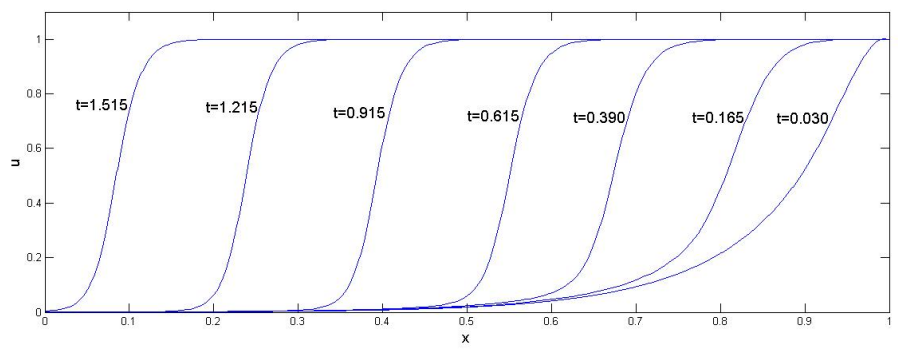

Figure 4: Evolution of Burgers equation solution $(0 \leq t \leq 1.515)$.

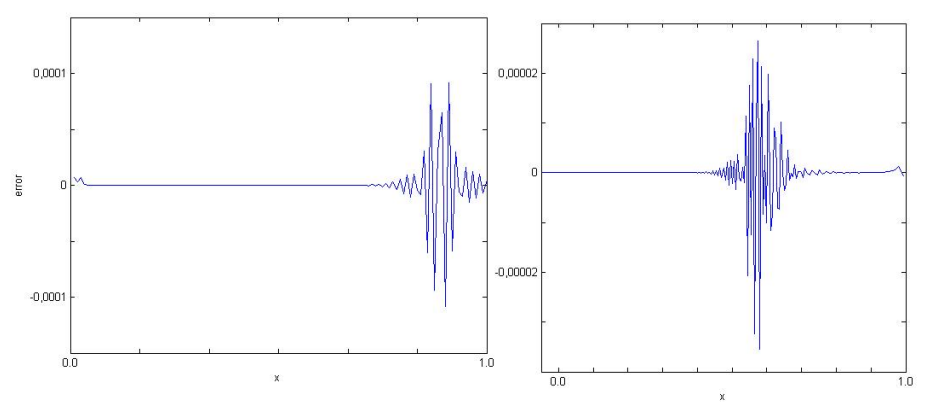

Figure 5: The approximation error versus $x$ (with knots in $k / 2^{7}, k=0, \ldots, 2^{7}$ ). Left for $t=0.15$ and right for $t=0.375$. 


\section{Conclusion}

In the present work an adaptive Wavelet-Galerkin method is proposed to solve partial differential equations of parabolic type. Numerical results reveal that the introduced technique is effective and convenient to solve these equations because it is easy to implement and yields the desired accuracy with low computational cost. In case of Burgers equation, the developed algorithm can track the moving fronts of the solutions and has the advantage of working well for small viscosity. We hope that the proposed method will be useful in more difficult and interesting cases, such as fluid mechanics problems.

\section{References}

[1] Bertoluzza, S.; Naldi, G. (1996) "A wavelet collocation method for the numerical solution of partial differential equations", Applied and Computational Harmonic Analysis 3(1): 1-9.

[2] Bindal, A.; Khinast, J.G.; Ierapetritou, M.G. (2003) "Adaptive multiscale solution of dynamical systems in chemical processes using wavelets", Computers and Chemical Engineering 27(1): 131-142.

[3] Burgers, J.M. (1948) "A mathematical model illustrating the theory of turbulence", Adv. Appl. Mech. 1: 171-199.

[4] Cammilleri, A; Serrano, E.P. (2001) "Spline multiresolution analysis on the interval", Latin American Applied Research 31(2): 65-71.

[5] Chui, C.K. (1992) An Introduction to Wavelets. Academic Press, New York.

[6] Ciarlet, P.G. (1978) The Finite Element Method for Elliptic Problems. North Holland, New York.

[7] Kumar, B V.; Mehra, M. (2005) "Wavelet-Taylor Galerkin method for the Burgers equation”, BIT Numerical Mathematics 45: 543-560.

[8] Kumar, V.; Mehra, M. (2007) "Cubic spline adaptive wavelet scheme to solve singularly perturbed reaction diffusion problems", International Journal of Wavelets, Multiresolution and Information Processing 5: 317331.

[9] Lin, E.B.; Zhou, X. (2001) "Connection coefficients on an interval and wavelet solutions of Burgers equation”, Journal of Computational and Applied Mathematics 135(1): 63-78. 
[10] Mallat, S.G. (2009) A Wavelet Tour of Signal Processing: The Sparse Way. Academic Press - Elsevier, MA EE.UU.

[11] Quraishi, S.M.; Gupta, R.; Sandeep, K. (2009) "Adaptive wavelet Galerkin solution of some elastostatics problems on irregularly spaced nodes", The Open Numerical Methods Journal 1: 20-26.

[12] Schöenberg, I.J. (1969) “Cardinal interpolation and spline functions”, Journal of Approximation Theory 2: 167-206.

[13] Schult, T.L.; Wyld, H.W. (1992) "Using wavelets to solve the Burgers equation: A comparative study”, Physical Review A 46(12): 7953-7958.

[14] Vampa, V.; Martín, M.T.; Serrano, E. (2010) "A hybrid method using wavelets for the numerical solution of boundary value problems on the interval", Appl. Math. Comput. 217(7): 3355-3367.

[15] Vampa, V. (2011) Desarrollo de Herramientas Basadas en la Transformada Wavelet para su Aplicación en la Resolución Numérica de Ecuaciones Diferenciales. Tesis de Doctorado en Matemática, Facultad de Ciencias Exactas, Universidad Nacional de La Plata, Argentina.

[16] Vampa, V.; Martín, M.T.; Serrano, E. (2012) "A new refinement WaveletGalerkin method in a spline local multiresolution analysis scheme for boundary value problems", Int. Journal of Wavelets, Multiresolution and Information Processing 11(2), 19 pp.

[17] Vasilyev, O.V.; Paolucci, S. (1996) "A dynamically adaptive multilevel wavelet collocation method for solving partial differential equations in a finite domain", Journal of Computational Physics 125(2): 498-512.

[18] Walnut, D.F. (2002) An Introduction to Wavelet Analysis. Applied and Numerical Harmonic Analysis Series, Birkhäuser, Boston.

[19] Whitham, G.B. (1974) Linear and Nonlinear Waves, Wiley, New York. 
